\title{
Bisphenol A Exacerbates Allergic Inflammation in an Ovalbumin-Induced Mouse Model of Allergic Rhinitis
}

\author{
Yunxiu Wang $\mathbb{D},{ }^{1}$ Zhiwei Cao $\mathbb{D}^{1},{ }^{1}$ He Zhao $\mathbb{D},{ }^{1}$ Yaoyao Ren $\mathbb{D},{ }^{1}$ Liying Hao $\mathbb{D}{ }^{2}$ \\ and Zhaowei Gu iD 1 \\ ${ }^{1}$ Department of Otolaryngology Head and Neck Surgery, Shengjing Hospital of China Medical University, Shenyang City, \\ 110004 Liaoning Province, China \\ ${ }^{2}$ Department of Pharmaceutical Toxicology, School of Pharmacy, China Medical University, Shenyang City, \\ 110122 Liaoning Province, China
}

Correspondence should be addressed to Liying Hao; hao_liying@126.com and Zhaowei Gu; guzw@sj-hospital.org

Received 3 June 2020; Revised 16 August 2020; Accepted 21 August 2020; Published 8 September 2020

Academic Editor: Massimo Ralli

Copyright (C) 2020 Yunxiu Wang et al. This is an open access article distributed under the Creative Commons Attribution License, which permits unrestricted use, distribution, and reproduction in any medium, provided the original work is properly cited.

Purpose. Bisphenol A (BPA) is found in many plastic products and is thus a common environmental endocrine disruptor. Plastic-related health problems, including allergic diseases, are attracting increasing attention. However, few experimental studies have explored the effect of BPA on allergic rhinitis (AR). We explore whether BPA was directly related to the allergic inflammation induced by ovalbumin (OVA) in AR mice. Methods. We first constructed OVA-induced mouse model, and after BPA administration, we evaluated nasal symptoms and measured the serum OVA-specific IgE levels by ELISA. Th2 and Treg-related cytokines of nasal mucosa were measured by cytometric bead array. Th2 and Treg-specific transcription factor levels were assayed by PCR. The proportions of $\mathrm{CD}^{+} \mathrm{CD}^{+} \mathrm{IL}^{-} 4^{+} \mathrm{Th} 2$ and $\mathrm{CD} 4^{+} \mathrm{Helios}^{+} \mathrm{Foxp} 3^{+} \mathrm{T}$ cells (Tregs) in spleen tissue were determined by flow cytometry. Results. Compared to OVA-only-induced mice, BPA addition increased nasal symptoms and serum OVA-specific IgE levels. OVA and BPA coexposure significantly increased IL-4 and IL-13 protein levels compared to those after OVA exposure alone. BPA plus OVA tended to decrease the IL-10 protein levels compared to those after OVA alone. Coexposure to OVA and BPA significantly increased the GATA-3-encoding mRNA level, and decreased the levels of mRNAs encoding Foxp3 and Helios, compared to those after OVA exposure alone. BPA increased the Th2 cell proportion, and decreased that of Tregs, compared to the levels with OVA alone. Conclusion. BPA exerted negative effects by exacerbating AR allergic symptoms, increasing serum OVA-specific IgE levels, and compromising Th2 and Treg responses.

\section{Introduction}

Allergic rhinitis (AR) is feature with nasal itching, sneezing, watery secretions, and congestion, reflecting the IgE-mediated mucosal inflammation driven by Th2 cells. AR affects over 500 million people worldwide [1,2]. Regulatory T cells (Tregs) play an important role in preventing Th2-mediated inappropriate responses to environmental allergens [3]. In recent years, the morbidity rate of AR has raised, especially among preschool children [4-6]. However, whether there is any role for external factors remains unclear. Bisphenol A (BPA) is a common environmental endocrine disruptor, being widely found in plastics. Humans come into contact with BPA via the skin and when consuming food and water packaged in plastic containing BPA [7]. BPA is an endocrine disruptor and may act as a weak estrogen; public health problems associated with BPA have attracted increasing attention [8]. BPA exposure during the perinatal or prenatal period exacerbated allergic sensitization and bronchial inflammation in asthma model $[9,10]$, and phthalates and BPA exacerbated atopic dermatitis in children [11]. Although epidemiological studies have not yet clearly shown that BPA increases the incidence 


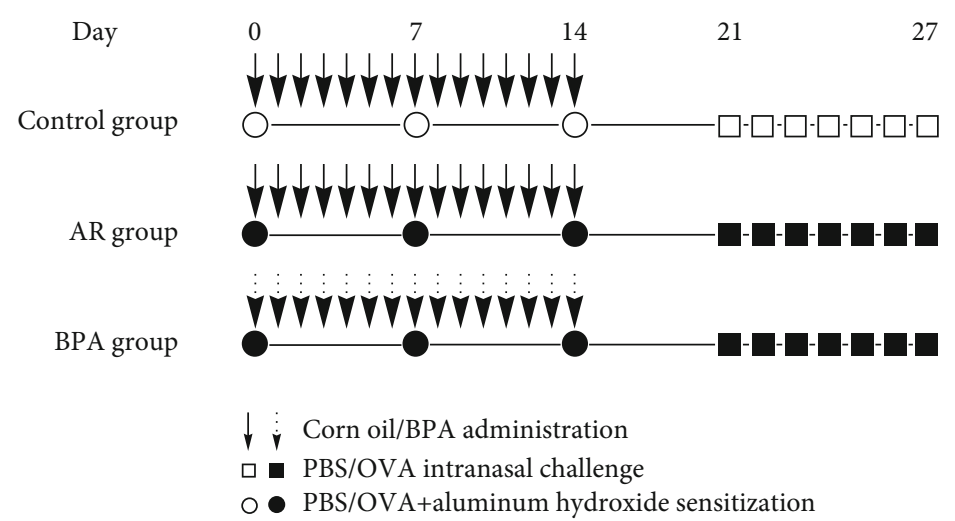

FIgURE 1: The experimental protocol. OVA sensitization was followed by OVA challenge to trigger AR development. Briefly, on days 0, 7, and 14 , mice in the OVA and BPA groups were sensitized with $2 \mathrm{mg}$ of aluminum hydroxide (100 $\mu \mathrm{L}$ of solution) and OVA (100 $\mu \mathrm{g})$ via intraperitoneal injection; control mice received PBS alone. Mice in the BPA group were subcutaneously injected with $0.5 \mathrm{mg} / \mathrm{kg} / \mathrm{day}$ BPA in corn oil on days $0-14$; the other two groups received only corn oil. The OVA and BPA groups were intranasally challenged with $100 \mu \mathrm{g}$ OVA in $20 \mu \mathrm{L}$ PBS on days 21-27. The control group received PBS alone. OVA: ovalbumin; AR: allergic rhinitis; BPA: bisphenol A; PBS: phosphate-buffered saline.

rates of allergy and asthma, it may enhance the risk of a Th2 response by altering immune cell function and cytokine production [12]. BPA combined with OVA exacerbated eosinophilia severity in the lungs of adult mice, perhaps by promoting a Th2-biased immune response [13]. As far as we know, few experimental studies have explored the effect of BPA on AR. Here, we explore whether BPA is directly related to the allergic inflammation induced by ovalbumin (OVA) in AR mice.

\section{Materials and Methods}

2.1. Reagents. RPMI 1640 was from Gibco, Carlsbad, CA. A Total RNA Extraction Kit was obtained from Solarbio (Beijing, China). BPA and OVA were obtained from SigmaAldrich (St. Louis, MO). A PrimeScript RT kit was ordered from Takara (Dalian, China). The APC-Cy7-CD3, FITCCD4, PE-IL-4, PE-Foxp3, APC-Helios, and CBA Flex Set were obtained from BD Biosciences (Franklin Lakes, NJ).

2.2. AR Murine Model and BPA Intervention. BALB/c mice ( 8 weeks, female) were purchased from Changsheng Biotechnology Co., Ltd., Liaoning, China. All mice were raised on an OVA-free diet and randomly assigned to control, AR, and BPA groups ( $n=10$ each). The AR model has been described previously [14]. On day 0 to day 14 , BPA mice were subcutaneously injected with $0.5 \mathrm{mg} / \mathrm{kg} / \mathrm{d}$ of BPA in corn oil; the other two groups received only corn oil. The experimental protocol, shown in Figure 1, was approved by the Ethics Committee of Shengjing Hospital.

2.3. Evaluation of Nasal Symptoms and Sample Collection. After the last intranasal OVA challenge, the numbers of sneezes and nose rubs over $15 \mathrm{~min}$ were recorded. Blood samples were collected from mice that were sacrificed under anesthesia; serum was obtained via centrifugation and stored at $-80^{\circ} \mathrm{C}$ prior to IgE detection. Nasal mucosal samples were stored for cytokine and quantitative real-time PCR (qRT-PCR) assays. Spleens were removed for detection of
$\mathrm{CD} 3^{+} \mathrm{CD} 4^{+} \mathrm{IL}_{-} 4^{+}$Th2 and $\mathrm{CD} 4^{+} \mathrm{Helios}^{+}$Foxp $^{+}$Tregs via flow cytometry.

2.4. Cytokine Measurements and Detection of OVA-Specific IgE. Nasal mucosa samples were crushed and centrifuged, and supernatant IL-4, IL-5, IL-13, and IL-10 levels were assayed using the CBA Flex Set. All samples underwent flow cytometry using the FACS Aria III instrument (BD Biosciences); the data were processed using FACSDiva and $\mathrm{BD}$ CBA software ver. 4.2 (BD Biosciences). Serum OVAspecific IgE levels were determined by ELISA kits (BioLegend).

2.5. qRT-PCR Analysis of Nasal Mucosal Samples. Total RNA was collected using the Total RNA Extraction Kit, and complementary DNA (cDNA) was synthesized via reverse transcription using the PrimeScript RT kit according to the manufacturer's instructions. qRT-PCR was performed using a Roche LightCycler 480 II system (Roche, Basel, Switzerland). The sequences of the PCR primers were listed in Table 1.

The relative expression of the three target genes was determined using the cycle threshold $\left(2^{-\triangle \triangle \mathrm{CT}}\right)$ method and normalized to the $\beta$-actin level.

2.6. Detection of $C D 3^{+} \mathrm{CD} 4^{+} \mathrm{IL}-4^{+}$Th2 and $\mathrm{CD} 4^{+} \mathrm{Helios}^{+} \mathrm{Foxp}^{+}$Tregs via Flow Cytometry. Splenic mononuclear cells were placed in tubes containing RPMI 1640 medium; $\mathrm{CD}^{+} \mathrm{CD} 4^{+} \mathrm{IL}-4^{+} \mathrm{Th} 2$ and $\mathrm{CD}^{+}{ }^{+}$Helios ${ }^{+}$Foxp $3{ }^{+}$Tregs were detected as described previously [15]. Stained cells were washed once and subjected to FACS Aria III flow cytometry (BD Biosciences); the results were analyzed using FlowJo software (ver. 7.6; TreeStar Inc., Ashland, OR).

2.7. Statistical Analysis. All data are expressed as means \pm SEM. One-way ANOVA was used to compare the groups. A $P$ value $<0.05$ was taken to indicate statistical significance. GraphPad Prism software (GraphPad Software Inc., La Jolla, CA) was used to statistically analyze and draw graphs. 
TABle 1: Primer sequences for quantitative real-time PCR.

\begin{tabular}{lcc}
\hline Gene & Sense & Antisense \\
\hline$\beta$-Actin & GCAGAAGGAGATTACTGCTCT & GCTGATCCACATCTGCTGGAA \\
Gata3 & TACCACCTATCCGCCCTATG & GCCTCGACTTACATCCGAAC \\
Foxp3 & GCCAAGCAGAAAGATGACAG & TTCCAGATGTTGTGGGTGAG \\
Helios & GGTACCGATGTGCTCTGCCT & TGTCCCTGTCACAGCAGAGC \\
\hline
\end{tabular}

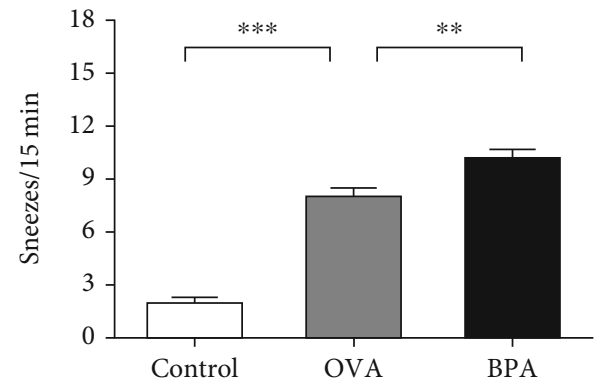

(a)

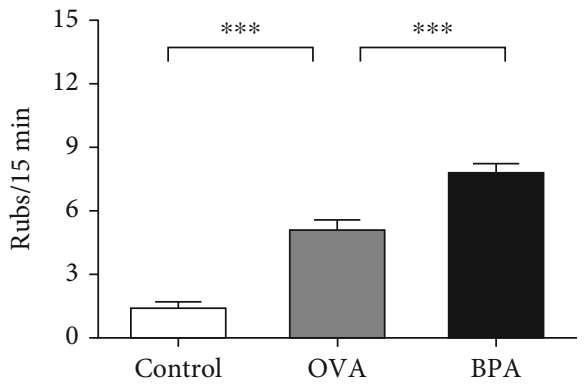

(b)

FIGURE 2: The numbers of (a) sneezes and (b) nasal rubs for each mouse were counted over 15 min from the last intranasal OVA challenge. Data are expressed as means \pm SEM; $n=10$ in each group. ${ }^{* *} P<0.01 ;{ }^{* * *} P<0.001$.

\section{Results}

3.1. Effects of BPA on OVA-Induced AR Nasal Symptom and OVA-Specific IgE Levels. Sneezing and nose scratching are the principal symptoms of AR; any effect of BPA on AR depends on the extent to which BPA affects these symptoms. We recorded numbers of sneezes and nose rubs in the three groups of mice over $15 \mathrm{~min}$ after the last intranasal OVA challenge. As shown in Figure 2, the OVA and BPA groups exhibited significantly more symptoms than the control $(P<0.05)$; OVA plus BPA mice (BPA group) showed more symptoms than the OVA-only group (OVA group) $(P<0.05)$. Serum OVA-specific IgE levels were significantly elevated in the OVA-induced group compared to the control group $(P<0.05)$ and were even higher in the OVA plus BPA group (Figure 3). BPA aggravated AR nasal symptoms and serum OVA-specific IgE levels.

3.2. Effects of BPA on Th2 Cytokine Levels in Nasal Mucosa. Th2-mediated cytokines cause inflammation in AR. The effects of BPA on nasal mucosal Th2 cytokine levels after the last intranasal OVA challenge were investigated. Compared to PBS, OVA increased IL-4, IL-5, and IL-13 protein levels $(P<0.05$, Figures $4(\mathrm{a})-4(\mathrm{c}))$. Compared to OVA alone, BPA further increased IL-4 and IL-13 protein levels $(P<0.05$, Figures 4(a) and 4(c)). BPA also increased the IL-5 levels, but not significantly (Figure 4(b)).

3.3. Effect of BPA on the Cytokine Levels of Tregs. As we knew, IL-10, a major cytokine of Tregs, plays an important role in the development of AR. Figure 4(d) shows that, compared to PBS, OVA reduced IL-10 protein levels $(P<0.05)$. BPA tended to further reduce the protein levels $(P<0.05)$.

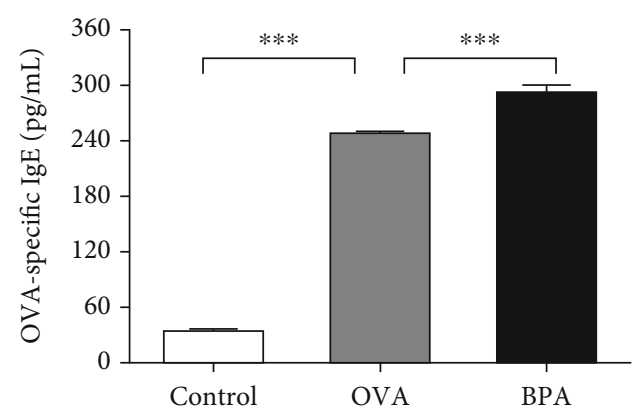

FIgURE 3: BPA increased the serum OVA-specific IgE levels, as shown by ELISA. Data are expressed as means \pm SEM; $n=5$ in each group. ${ }^{* * *} P<0.001$.

\subsection{Effect of BPA on Th2 Cell-Specific Transcription Factors.} The effect of BPA on the levels of a Th2 cell-specific transcription factor (GATA-3) was evaluated. Compared to PBS, OVA increased the levels of mRNA encoding GATA$3(P<0.05$, Figure 5(a)). BPA further increased the levels $(P<0.05)$.

3.5. Effect of BPA on Treg-Specific Transcription Factor Levels. Foxp3 is the most specific marker of Tregs; Helios status is helpful for identifying Treg subsets showing consistent suppressive activity [16]. We measured the levels of mRNA encoding Foxp3 and Helios. Figures 5(b) and 5(c) show that, compared to PBS, OVA reduced these levels; BPA further reduced the levels $(P<0.05)$.

3.6. Effect of BPA on the Proportions of Th2 Cells. An imbalance among the $\mathrm{CD} 4^{+} \mathrm{Th}$ cell subsets, particularly Th2 cells, triggers and maintains allergic responses [17]. We assessed the effect of BPA on the proportions of Th2 cell (Figure 6). 


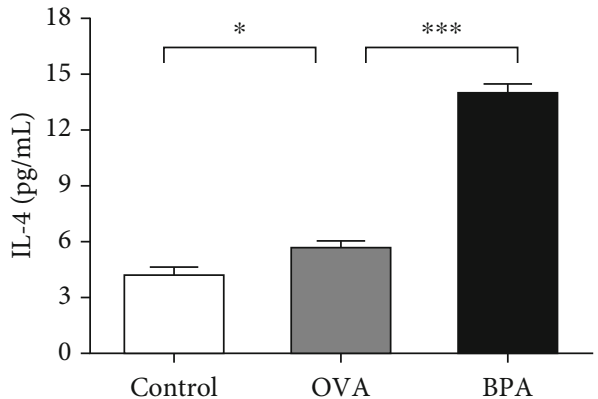

(a)

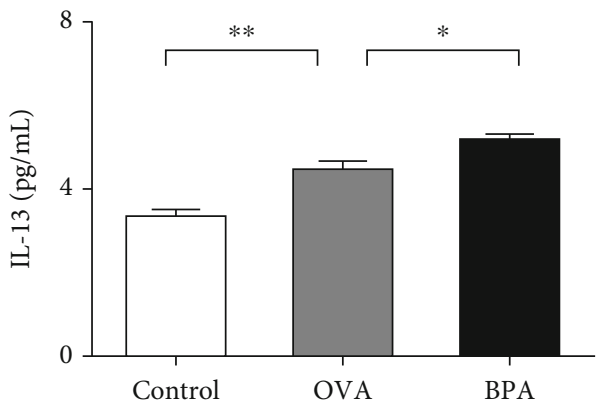

(c)

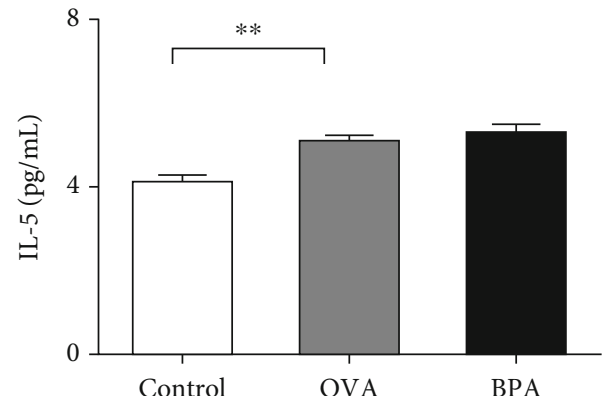

(b)

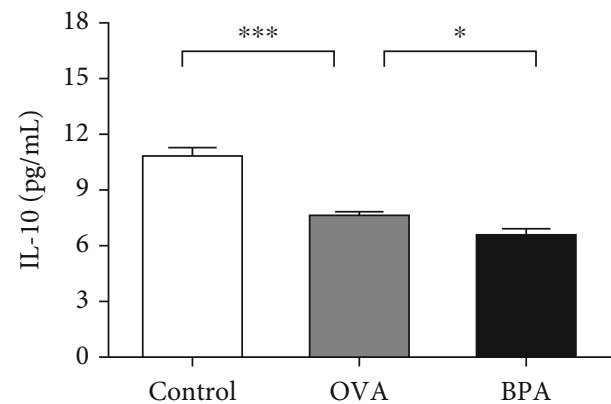

(d)

Figure 4: BPA increased the levels of Th2 cell effector cytokines and repressed cytokine expression by Tregs in the nasal mucosa. The protein expression levels of (a) IL-4, (b) IL-5, (c) IL-13, and (d) IL-10 were measured using the CBA Flex Set (BD Biosciences). Data are expressed as means \pm SEM; $n=5$ in each group. ${ }^{*} P<0.05 ;{ }^{* *} P<0.01 ;{ }^{* * *} P<0.001$.

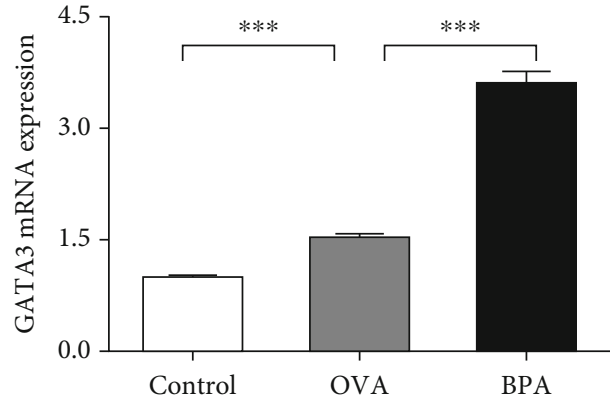

(a)

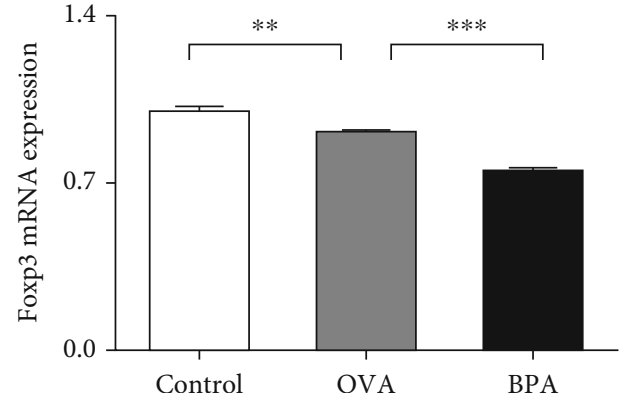

(b)

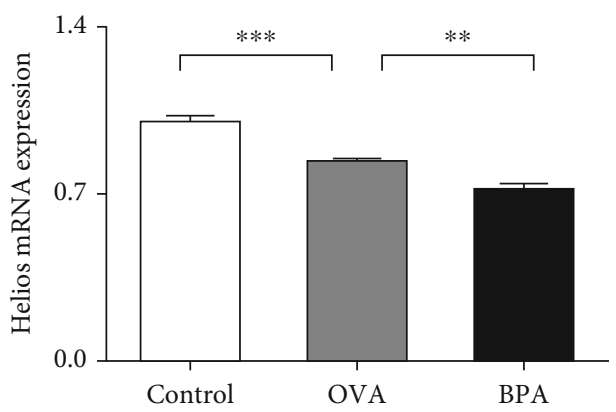

(c)

Figure 5: BPA increased the levels of the Th2 cell-specific transcription factor GATA-3 and reduced those of the Treg-specific factors Foxp3 and Helios. RT-PCR data on (a) GATA-3, (b) Foxp3, and (c) Helios mRNA expression levels in nasal mucosa. Data are expressed as means \pm SEM; $n=5$ in each group. ${ }^{* *} P<0.01 ;{ }^{* * *} P<0.001$.

OVA increased the proportions of $\mathrm{CD} 3^{+} \mathrm{CD} 4^{+} \mathrm{IL}-4^{+} \mathrm{Th} 2$ cells compared to control mice; BPA further increased the proportions of these cells $(P<0.05)$.
3.7. Effect of BPA on the Proportion of Tregs. Tregs modulate the immune system and maintain tolerance to self-antigens [18]. We measured the proportion of $\mathrm{CD} 4^{+} \mathrm{Helios}^{+} \mathrm{Foxp}^{+}$ 


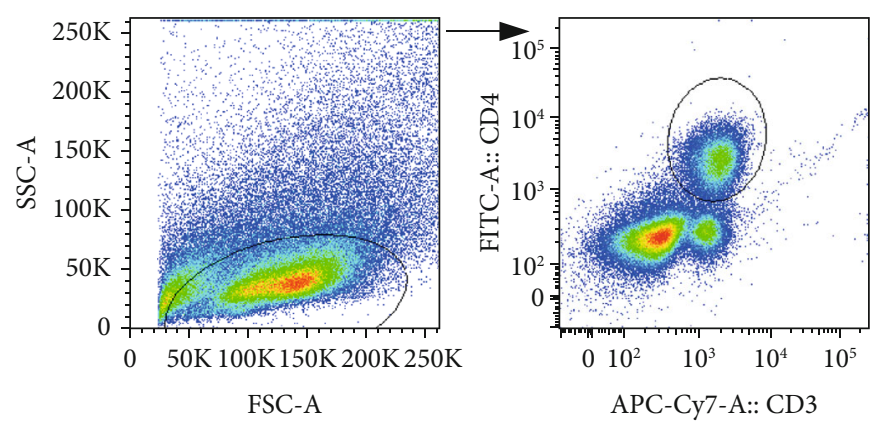

(a)
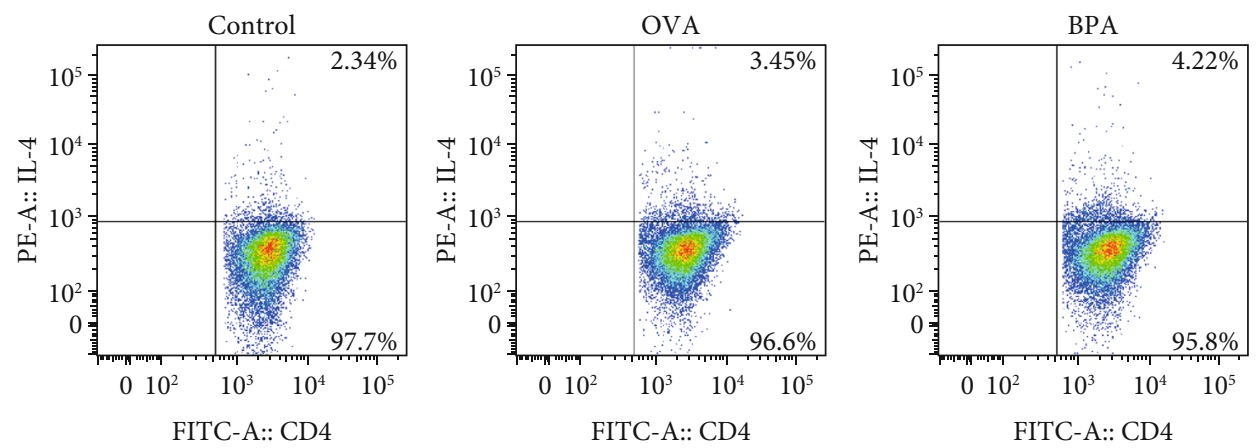

(b)

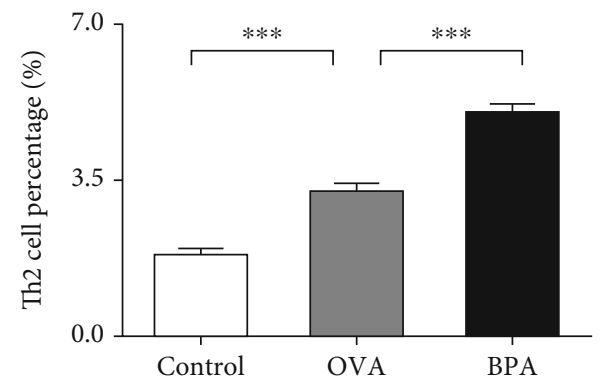

(c)

FIGURE 6: Flow cytometry showed that BPA increased the proportions of Th2 cells in the spleens of mice with OVA-induced AR. CD3 ${ }^{+} \mathrm{CD} 4^{+}$ $\mathrm{T}$ cell subgroup (a). Representative staining of $\mathrm{CD} 3^{+} \mathrm{CD} 4^{+} \mathrm{IL}-4^{+} \mathrm{Th} 2$ cells of each group. The numbers in the upper right quadrants are the proportions of Th2 cells (b). The statistical data (c). Data are expressed as means \pm SEM; $n=5$ in each group. ${ }^{* * *} P<0.001$.

Tregs via flow cytometry; compared to PBS, OVA decreased the proportions of these cells $(P<0.05$, Figure 7$)$. BPA further decreased the proportions of the cells compared to OVA alone $(P<0.05)$.

\section{Discussion}

We used an established OVA-induced AR murine model to explore whether BPA affected allergic reactions. Nasal symptoms were exacerbated; meanwhile, OVA-specific IgE levels were increased, in BPA plus OVA-treated AR mice. BPA increased the proportions of Th2 cells, as well as the mRNA levels of GATA-3- and Th2-related cytokines IL-4 and IL13. BPA downregulated Treg cells, the mRNA levels of Helios and Foxp3, and Treg-related cytokine IL-10. Many previous studies have focused on off-target effects of BPA [19]; we evaluated the direct effects of BPA on allergic inflammation. Mice with AR received BPA prior to OVA challenge; we sought to establish if there was a direct relationship between the allergic response and BPA. Allergens activate the binding of IgE to FceRI located on the surface of eosinophils; chemical mediators are then released, triggering clinical symptoms of allergy [17]. AR symptoms are thus attributable to IgEmediated inflammation of the nasal mucosa. We found that BPA exposure significantly increased allergic symptoms and serum OVA-specific IgE levels; thus, BPA directly affected experimental AR.

$\mathrm{AR}$ is a common disorder caused by an inappropriate Th2-mediated immune response to environmental antigens [20]. Th2 cells secrete specific cytokines, including IL-4, IL-5, and IL-13 [21-23], which are important drivers of AR immunopathology [24]. IL-4 promotes T cell activation and differentiation into Th2 cells; IL-4 and IL-13 play roles in B cell differentiation and $\mathrm{IgE}$ and mucus production in the airway [25-27]. IL-5 is locally produced at sites of allergic inflammation and recruits eosinophils from the bone 


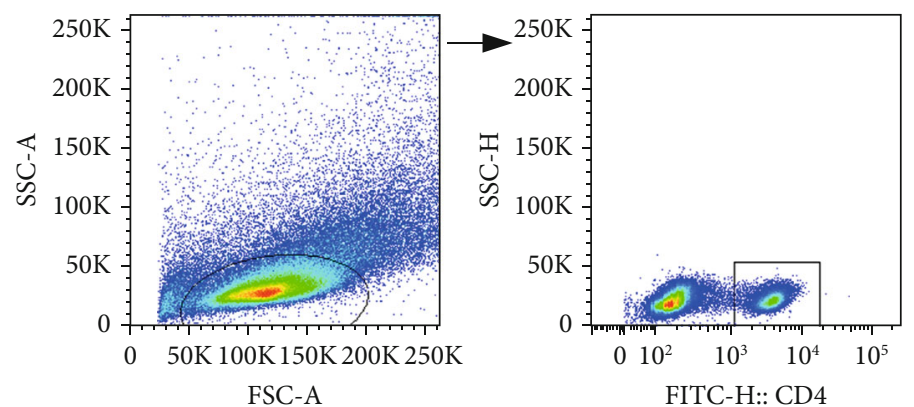

(a)
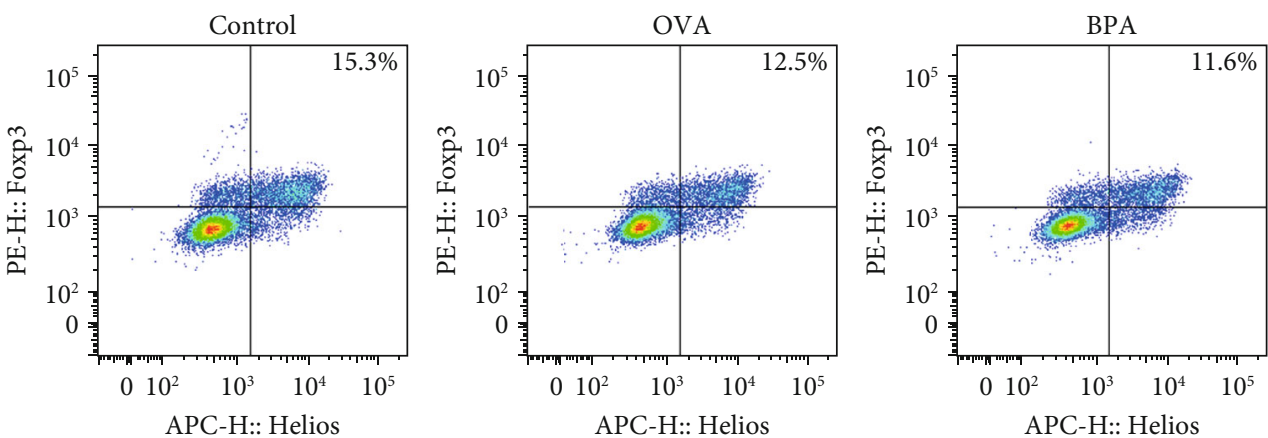

(b)

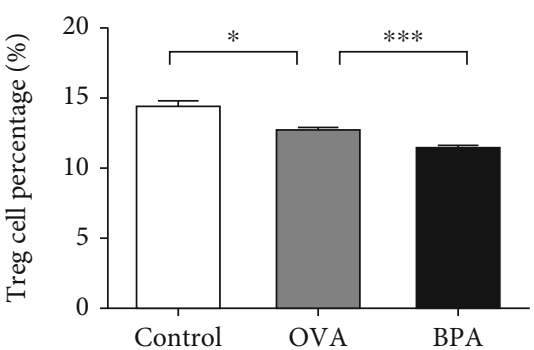

(c)

Figure 7: Flow cytometry showed that BPA reduced the proportion of Tregs in spleens of mice with OVA-induced AR. CD4 ${ }^{+}$T cells (a). Representative staining of $\mathrm{CD}^{+} \mathrm{Helios}^{+} \mathrm{Foxp} 3^{+}$Tregs from each group. The numbers in the upper right quadrants are the proportions of Tregs (b). The statistical data (c). Data are expressed as means \pm SEM; $n=5$ per group. ${ }^{*} P<0.05 ;{ }^{* * *} P<0.001$.

marrow; these cells contribute to AR injury by releasing cytotoxic granular proteins $[22,28]$. The GATA-3 transcription factor is specific to Th2 cells. It promotes the production of IL-4, IL-5, and IL-13; induces Th0 to differentiate into Th2; and inhibits Th1 cell differentiation [29]. We explored the effects of BPA on Th2 cells by measuring the proportions of $\mathrm{CD} 3^{+} \mathrm{CD} 4^{+} \mathrm{IL}-4^{+} \mathrm{Th} 2$ cells, as well as the protein levels of Th2-related cytokines, and the mRNA level of GATA-3. BPA significantly increased expression of all of these cells and factors. Our results are similar to those of Yanagisawa [30]; OVA and BPA coexposure increased the mouse lung levels of mRNAs encoding IL-4, IL-5, and IL13 compared to those after OVA exposure alone [30]. We found that the IL-5 level in the BPA mice was somewhat higher than in the OVA ones; however, the difference was not significant, perhaps because of the small sample sizes ( $n=5$ /group).

Treg prevents inappropriate Th2 responses to environmental allergens $[3,31]$. Th2 cells secrete inhibitory cyto- kines, including IL-10, that induce Treg formation from naive $\mathrm{T}$ cells and inhibit the development of other types of immune cells [32]. Foxp3 is a Treg-related transcription factor, and Helios is a marker of Treg activation [33, 34]. The effects of BPA on the Treg response were evaluated by measuring the proportion of Tregs, IL-10 protein levels, and Helios and Foxp3 mRNA levels. Compared to OVA alone, coexposure to OVA and BPA reduced the Treg response (i.e., the proportion of Tregs, IL-10 protein levels, Helios and Foxp3 mRNA levels). Previous studies showed that BPA reduced the Tregs proportion, thus compromising the immune system; the Th1/Th2 ratio changed and disease developed $[35,36]$.

BPA is a common environmental endocrine disruptor, which can disrupt the human endocrine, reproductive, and immune systems through cell signaling pathways, and can increase the risk for certain diseases, including obesity, cardiovascular disease, brain disease, asthma, and even cancer $[36,37]$. The signaling pathways affected by BPA include 
signal transducer and activator of transcription 3 (STAT3), early growth response gene-2, NF- $\kappa \mathrm{B}$, and ERK1/2 [38-43].

Previous AR immune cellular signal pathway studies have mainly focused on the influence of the STAT family on Th differentiation [44]. STAT family members include STAT1, STAT2, STAT3, STAT4, STAT5A, STAT5b, and STAT6 [45]. STAT1 and STAT4 are the key factors of IFN- $\gamma$ signaling and IL-12 signaling, respectively, and both are critical for Th1 polarization [46]. However, STAT6 can inhibit Th1 polarization, which is an important factor in Th2 signal transduction [46]. STAT5 can regulate the differentiation of Treg cells by regulating Foxp3 expression [47]. STAT3 is an essential transcription factor for Th17 differentiation and Treg inhibition $[45,48]$. In addition, it can promote the development of Th2 cells under the background of STAT6 signaling [49].

STAT3 is activated by immune cytokines and endocrinedisrupting chemicals, including BPA [50-52]. Therefore, we speculated that STAT3 may be activated after exposure to $\mathrm{BPA}$, the Th2 response is enhanced, and Treg reaction is inhibited through the STAT3 signaling pathway, further aggravating AR inflammation.

Our previous studies confirmed the effects of the environmental hormone nonylphenol on $\mathrm{AR}$, and showed that nonylphenol can aggravate Th2-associated immune reactions in an AR mouse model [14]. The present study focused on BPA. However, a comprehensive analysis is required to determine the combined actions of environmental endocrine disruptors.

\section{Conclusion}

Our findings provide evidence of the negative effects of BPA in an OVA-induced AR mouse model. BPA can exacerbate AR allergic symptoms, increase serum levels of OVAspecific IgE, and compromise Th2 and Treg responses. Our results serve as a warning regarding the adverse effects of BPA in adult AR.

\section{Data Availability}

The data used to support the findings of this study are available from the corresponding author upon request.

\section{Conflicts of Interest}

The authors declare that they have no competing interests.

\section{Authors' Contributions}

YW, ZG, HZ, ZC, and LH conceived and designed the experiments. ZG and YW performed the experiments. HZ, ZC, YR, and LH analyzed the data. ZG and YW wrote the paper. Authors read and approved the final manuscript.

\section{Acknowledgments}

This research was supported by the National Natural Science Foundation of China (Grant No. 21677030) and the
Liaoning Province Ministry of Education Scientific Study Project (Grant No. JC2019010).

\section{References}

[1] S. Akasaki, K. Matsushita, Y. Kato et al., "Murine allergic rhinitis and nasal Th2 activation are mediated via TSLP- and IL-33-signaling pathways," International Immunology, vol. 28, no. 2, pp. 65-76, 2016.

[2] A. N. Greiner, P. W. Hellings, G. Rotiroti, and G. K. Scadding, "Allergic rhinitis," Lancet, vol. 378, no. 9809, pp. 2112-2122, 2011.

[3] Q. Zeng, W. Liu, R. Luo, and G. Lu, "MicroRNA-181a and microRNA-155 are involved in the regulation of the differentiation and function of regulatory $\mathrm{T}$ cells in allergic rhinitis children," Pediatric Allergy and Immunology, vol. 30, no. 4, pp. 434-442, 2019.

[4] J. Bousquet, N. Khaltaev, A. A. Cruz et al., "Allergic rhinitis and its impact on asthma (ARIA) 2008 update (in collaboration with the World Health Organization, GA(2)LEN and AllerGen)," Allergy, vol. 63, Suppl 86, pp. 8-160, 2008.

[5] P. W. Hellings and J. L. Ceuppens, "Mouse models of global airway allergy: what have we learned and what should we do next?," Allergy, vol. 59, no. 9, pp. 914-919, 2004.

[6] J. Hellgren, A. Cervin, S. Nordling, A. Bergman, and L. O. Cardell, "Allergic rhinitis and the common cold-high cost to society," Allergy, vol. 65, no. 6, pp. 776-783, 2010.

[7] C. Kubwabo, I. Kosarac, B. Stewart, B. R. Gauthier, K. Lalonde, and P. J. Lalonde, "Migration of bisphenol A from plastic baby bottles, baby bottle liners and reusable polycarbonate drinking bottles," Food Additives \& Contaminants. Part A, Chemistry, Analysis, Control, Exposure \& Risk Assessment, vol. 26, no. 6, pp. 928-937, 2009.

[8] C. Liao and K. Kannan, "Concentrations and profiles of bisphenol A and other bisphenol analogues in foodstuffs from the United States and their implications for human exposure," Journal of Agricultural and Food Chemistry, vol. 61, no. 19, pp. 4655-4662, 2013.

[9] Y. Nakajima, R. M. Goldblum, and T. Midoro-Horiuti, "Fetal exposure to bisphenol A as a risk factor for the development of childhood asthma: an animal model study," Environmental Health, vol. 11, no. 1, 2012.

[10] T. Midoro-Horiuti, R. Tiwari, C. S. Watson, and R. M. Goldblum, "Maternal bisphenol A exposure promotes the development of experimental asthma in mouse pups," Environmental Health Perspectives, vol. 118, no. 2, pp. 273-277, 2010.

[11] E. H. Kim, B. H. Jeon, J. Kim et al., "Exposure to phthalates and bisphenol A are associated with atopic dermatitis symptoms in children: a time-series analysis," Environmental Health, vol. 16, no. 1, p. 24, 2017.

[12] L. Robinson and R. Miller, "The impact of bisphenol A and phthalates on allergy, asthma, and immune function: a review of latest findings," Curr Environ Health Rep., vol. 2, no. 4, pp. 379-387, 2015.

[13] M. He, T. Ichinose, S. Yoshida et al., "Exposure to bisphenol A enhanced lung eosinophilia in adult male mice," Allergy, Asthma and Clinical Immunology, vol. 12, no. 1, 2016.

[14] Y. X. Wang, Z. W. Gu, Z. W. Cao, and L. Y. Hao, "Nonylphenol can aggravate allergic rhinitis in a murine model by regulating important Th cell subtypes and their associated 
cytokines," International Immunopharmacology, vol. 70, pp. 260-267, 2019.

[15] Y. X. Wang, Z. W. Gu, and L. Y. Hao, "The environmental hormone nonylphenol interferes with the therapeutic effects of G protein-coupled estrogen receptor specific agonist G-1 on murine allergic rhinitis," International Immunopharmacology, vol. 78, p. 106058, 2020.

[16] Y. X. Wang, Z. W. Gu, and Z. W. Cao, "Difference between CD25+Tregs and Helios+Tregs in a murine model of allergic rhinitis," Brazilian Journal of Otorhinolaryngology, 2020.

[17] J. Wang, Y. Shen, C. Li et al., "IL-37 attenuates allergic process via STAT6/STAT3 pathways in murine allergic rhinitis," International Immunopharmacology, vol. 69, pp. 27-33, 2019.

[18] J. Li, B. Wang, Y. Luo, Y. Bian, and R. Wang, "Effect of artemisinin and neurectomy of pterygoid canal in ovalbumininduced allergic rhinitis mouse model," Allergy, Asthma and Clinical Immunology, vol. 14, no. 1, 2018.

[19] R. Tajiki-Nishino, E. Makino, Y. Watanabe, H. Tajima, M. Ishimota, and T. Fukuyama, "Oral administration of bisphenol A directly exacerbates allergic airway inflammation but not allergic skin inflammation in mice," Toxicological Sciences, vol. 165, no. 2, pp. 314-321, 2018.

[20] M. Wang, Z. Gu, J. Yang, H. Zhao, and Z. Cao, "Changes among TGF- $\beta 1+$ Breg cells and helper $\mathrm{T}$ cell subsets in a murine model of allergic rhinitis with prolonged OVA challenge," International Immunopharmacology, vol. 69, pp. 347357, 2019.

[21] H. Yssel and H. Groux, "Characterization of T cell subpopulations involved in the pathogenesis of asthma and allergic diseases," International Archives of Allergy and Immunology, vol. 121, no. 1, pp. 10-18, 2000.

[22] A. O. Eifan and S. R. Durham, "Pathogenesis of rhinitis," Clinical and Experimental Allergy, vol. 46, no. 9, pp. 1139-1151, 2016.

[23] P. H. Howarth, M. Salagean, and D. Dokic, "Allergic rhinitis: not purely a histamine-related disease," Allergy, vol. 55, Suppl 64, pp. 7-16, 2000.

[24] C. Sanden, M. Mori, P. Jogdand et al., "Broad Th2 neutralization and anti-inflammatory action of pentosan polysulfate sodium in experimental allergic rhinitis," Immunity, Inflammation and Disease, vol. 5, no. 3, pp. 300-309, 2017.

[25] J. J. Shim, K. Dabbagh, I. F. Ueki et al., "IL-13 induces mucin production by stimulating epidermal growth factor receptors and by activating neutrophils," American Journal of Physiology. Lung Cellular and Molecular Physiology, vol. 280, no. 1, pp. L134-L140, 2001.

[26] A. J. Frew, "The immunology of respiratory allergies," Toxicology Letters, vol. 86, no. 2-3, pp. 65-72, 1996.

[27] R. D. May and M. Fung, "Strategies targeting the IL-4/IL-13 axes in disease," Cytokine, vol. 75, no. 1, pp. 89-116, 2015.

[28] C. A. Ahlstrom-Emanuelsson, L. Greiff, M. Andersson, C. G. Persson, and J. S. Erjefält, "Eosinophil degranulation status in allergic rhinitis: observations before and during seasonal allergen exposure," The European Respiratory Journal, vol. 24, no. 5, pp. 750-757, 2004.

[29] R. Yagi, J. Zhu, and W. E. Paul, “An updated view on transcription factor GATA3-mediated regulation of Th1 and Th2 cell differentiation," International Immunology, vol. 23, no. 7, pp. 415-420, 2011.

[30] R. Yanagisawa, E. Koike, T. T. Win-Shwe, and H. Takano, "Oral exposure to low dose bisphenol A aggravates allergic airway inflammation in mice," Toxicology Reports, vol. 6, pp. 1253-1262, 2019.

[31] S. Yu, B. Han, S. Liu et al., "Derp1-modified dendritic cells attenuate allergic inflammation by regulating the development of T helper type1(Th1)/Th2 cells and regulatory T cells in a murine model of allergic rhinitis," Molecular Immunology, vol. 90, pp. 172-181, 2017.

[32] F. Meiler, J. Zumkehr, S. Klunker, B. Rückert, C. A. Akdis, and M. Akdis, "In vivo switch to IL-10-secreting T regulatory cells in high dose allergen exposure," The Journal of Experimental Medicine, vol. 205, no. 12, pp. 2887-2898, 2008.

[33] S. B. Wang, Y. Q. Deng, J. Ren, B. K. Xiao, Z. Chen, and Z. Z. Tao, "Lactoferrin administration into the nostril alleviates murine allergic rhinitis and its mechanisms," Scandinavian Journal of Immunology, vol. 78, no. 6, pp. 507-515, 2013.

[34] A. S. S. Khaja, S. M. Toor, H. El Salhat, B. R. Ali, and E. Elkord, "Intratumoral FoxP3+Helios+ Regulatory T Cells Upregulating Immunosuppressive Molecules Are Expanded in Human Colorectal Cancer," Frontiers in Immunology, vol. 8, 2017.

[35] J. A. Rogers, L. Metz, and V. W. Yong, "Review: endocrine disrupting chemicals and immune responses: a focus on bisphenol-A and its potential mechanisms," Molecular Immunology, vol. 53, no. 4, pp. 421-430, 2013.

[36] J. Xu, G. Huang, and T. L. Guo, "Developmental bisphenol A exposure modulates immune-related diseases," Toxics., vol. 4, no. 4, p. 23, 2016.

[37] O. Yang, H. L. Kim, J. I. Weon, and Y. R. Seo, "Endocrine-disrupting chemicals: review of toxicological mechanisms using molecular pathway analysis," J Cancer Prev., vol. 20, no. 1, pp. 12-24, 2015.

[38] F. M. Huang, Y. C. Chang, S. S. Lee, M. L. Yang, and Y. H. Kuan, "Expression of pro-inflammatory cytokines and mediators induced by bisphenol A via ERK-NF $\kappa \mathrm{B}$ and JAK1/2STAT3 pathways in macrophages," Environmental Toxicology, vol. 34, no. 4, pp. 486-494, 2019.

[39] Y. Liu, C. Mei, H. Liu et al., "Modulation of cytokine expression in human macrophages by endocrine-disrupting chemical bisphenol-A," Biochemical and Biophysical Research Communications, vol. 451, no. 4, pp. 592-598, 2014.

[40] N. Couleau, J. Falla, A. Beillerot et al., "Effects of endocrine disruptor compounds, alone or in combination, on human macrophage-like THP-1 cell response," PLoS One, vol. 10, no. 7, article e0131428, 2015.

[41] J. Y. Kim and H. G. Jeong, "Down-regulation of inducible nitric oxide synthase and tumor necrosis factor-alpha expression by bisphenol A via nuclear factor-kappaB inactivation in macrophages," Cancer Letters, vol. 196, no. 1, pp. 69-76, 2003.

[42] S. H. Lam, M. M. Hlaing, X. Zhang et al., “Toxicogenomic and phenotypic analyses of bisphenol-A early-life exposure toxicity in zebrafish," PLoS One, vol. 6, no. 12, article e28273, 2011.

[43] K. S. Saili, S. C. Tilton, K. M. Waters, and R. L. Tanguay, "Global gene expression analysis reveals pathway differences between teratogenic and non-teratogenic exposure concentrations of bisphenol A and $17 \beta$-estradiol in embryonic zebrafish," Reproductive Toxicology, vol. 38, pp. 89-101, 2013.

[44] R. Goswami and M. H. Kaplan, "STAT transcription factors in T cell control of health and disease," International Review of Cell and Molecular Biology, vol. 331, pp. 123-180, 2017. 
[45] F. Seif, M. Khoshmirsafa, H. Aazami, M. Mohsenzadegan, G. Sedighi, and M. Bahar, "The role of JAK-STAT signaling pathway and its regulators in the fate of T helper cells," Cell Communication and Signaling: CCS, vol. 15, no. 1, p. 23, 2017.

[46] J. J. O'Shea and P. J. Murray, "Cytokine signaling modules in inflammatory responses," Immunity, vol. 28 , no. 4, pp. 477487, 2008.

[47] M. A. Burchill, J. Yang, C. Vogtenhuber, B. R. Blazar, and M. A. Farrar, "IL-2 receptor $\beta$-Dependent STAT5 activation is required for the development of Foxp3+Regulatory T cells," Journal of Immunology, vol. 178, no. 1, pp. 280-290, 2006.

[48] P. Zhao, J. Li, Y. Tian et al., "Restoring Th17/Treg balance via modulation of STAT3 and STAT5 activation contributes to the amelioration of chronic obstructive pulmonary disease by Bufei Yishen formula," Journal of Ethnopharmacology, vol. 217, pp. 152-162, 2018.

[49] G. L. Stritesky, R. Muthukrishnan, S. Sehra et al., "The transcription factor STAT3 is required for T helper 2 cell development," Immunity, vol. 34, no. 1, pp. 39-49, 2011.

[50] H. Lee, S. K. Pal, K. Reckamp, R. A. Figlin, and H. Yu, "STAT3: a target to enhance antitumor immune response," Current Topics in Microbiology and Immunology, vol. 344, pp. 41-59, 2011.

[51] C. J. Queval, O.-R. Song, N. Deboosère et al., "STAT3 represses nitric oxide synthesis in human macrophages upon mycobacterium tuberculosis infection," Scientific Reports, vol. 6, no. 1, 2016.

[52] Y. Sekine, T. Yamamoto, T. Yumioka, S. Imoto, H. Kojima, and T. Matsuda, "Cross-talk between endocrine-disrupting chemicals and cytokine signaling through estrogen receptors," Biochemical and Biophysical Research Communications, vol. 315, no. 3, pp. 692-698, 2004. 